\title{
The Yeast Metabolic Cycle: Insights into the Life of a Eukaryotic Cell
}

\author{
B.P. TU AND S.L. MCKNight \\ Department of Biochemistry, University of Texas Southwestern Medical Center, Dallas, \\ Texas 75390-9038
}

\begin{abstract}
The budding yeast Saccharomyces cerevisiae undergoes robust oscillations in oxygen consumption during continuous growth under nutrient-limited conditions. Comprehensive microarray studies reveal that more than half of the yeast genome is expressed periodically as a function of these respiratory oscillations, thereby specifying an extensively orchestrated program responsible for regulating numerous cellular outputs. Here, we summarize the logic of the yeast metabolic cycle (YMC) and highlight additional cellular processes that are predicted to be compartmentalized in time. Certain principles of temporal orchestration as seen during the YMC might be conserved across other biological cycles.
\end{abstract}

\section{INTRODUCTION}

The budding yeast $S$. cerevisiae has been known for decades to be capable of exhibiting various modes of oscillatory behavior, both in cell-free extracts and during continuous growth (Chance et al. 1964; von Meyenburg 1969; Satroutdinov et al. 1992; Richard 2003; Klevecz et al. 2004). We recently studied a continuous culture system that revealed a robust approximately $4-5$-hour YMC that occurs under nutrient-limited growth conditions $(\mathrm{Tu}$ et al. 2005). When prototrophic yeast cells are grown in culture to a high density, starved for a short period, and then continuously fed low concentrations of glucose, the population of cells becomes highly synchronized and undergoes robust oscillations of oxygen consumption (Fig. 1). During these oscillations, cells proceed through phases where they rapidly consume oxygen, followed by longer phases where they consume much less oxygen (Fig. 1). The period length of these metabolic cycles under such growth conditions is typically about 4-5 hours, but it can vary depending on the rate of glucose addition (Tu et al. 2005). Such cycles persist as long as continuous concentrations of glucose are supplied to the cells.

Remarkably, more than half of yeast genes $(\sim 57 \%)$ are expressed periodically as a precise function of the YMC (Tu et al. 2005). Genes that encode proteins with a common function often display similar temporal expression patterns, and different classes of genes are up-regulated at entirely different temporal windows of the YMC. Moreover, genes that encode proteins having functions that are associated with energy and metabolism tend to be expressed with exceptionally precise and robust periodicity, suggesting that these cycles are intrinsically metabolic (Tu et al. 2005).

Analysis of the temporal gene expression profiles revealed three superclusters of gene expression, thereby defining three primary phases of the YMC: OX (oxidative, respiratory), RB (reductive, building), and RC (reductive, charging) (Fig. 1) (Tu et al. 2005). Different categories of genes peak during each phase, and cells

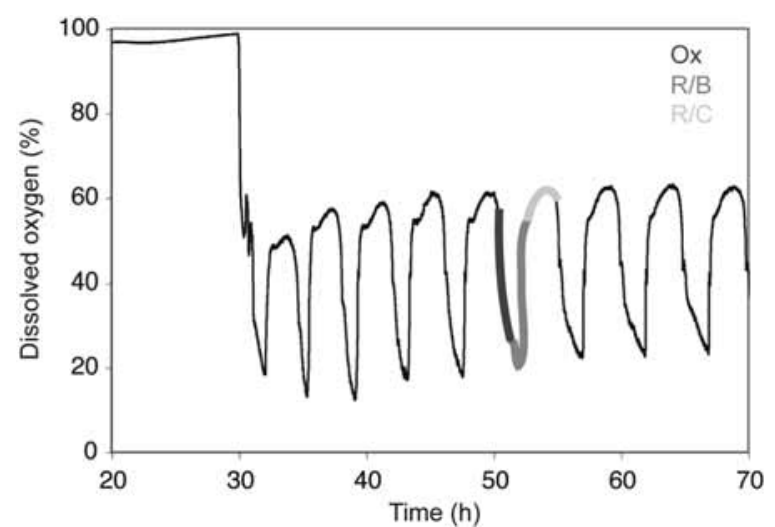

Figure 1. The yeast metabolic cycle. During continuous culture growth, budding yeast exhibit robust metabolic cycles as measured by oxygen consumption. These cycles are composed of three major phases: OX (oxidative), RB (reductive, building), and RC (reductive charging). Shown are approximately 4-5hour metabolic cycles exhibited by the wild-type diploid strain CEN.PK upon continuous feeding of glucose.

successively pass through each of the three phases of the YMC in every cycle. The temporal gene expression data predict that the YMC controls precisely when certain cellular and metabolic events occur (e.g., respiration, mitochondria biogenesis, ribosome biogenesis, cell division, fatty acid oxidation, glycolysis, and autophagy), indicating that key cellular processes in a simple eukaryotic cell are compartmentalized in time ( $\mathrm{Tu}$ et al. 2005). Such temporal compartmentalization might provide a means for the cell to perform a multitude of metabolic processes in a more coordinated and efficient fashion and help minimize futile reactions, especially under nutrient-limited growth environments. Periodic gene expression patterns prototypical of both the circadian cycle and the YMC signify that organisms have developed a number of sophisticated strategies to take advantage of the dimension of time. 


\section{THREE METABOLIC PHASES IN THE LIFE OF A YEAST CELL}

Inspection of the genes that are maximally expressed in each phase of the YMC has provided insight into the cellular outputs and events that occur during particular temporal windows of the YMC. The OX phase is relatively brief compared to the reductive $\mathrm{RB}$ and $\mathrm{RC}$ phases and is characterized by a period of intense respiration (Fig. 1). Many ribosomal genes and amino acid biosynthetic genes are induced in the OX phase, suggesting that this phase is dedicated to establishing the protein synthesis machinery for growth and the preparation of cell division ( $\mathrm{Tu}$ et al. 2005). Both ribosome and amino acid biosynthesis, which are highly energetically demanding, might be coordinated with the OX phase in order to benefit from copious amounts of ATP that are produced from respiration. Many genes encoding components of the nucleolus, small nuclear RNAs (snRNAs), and transfer RNA (tRNA) synthetases are also up-regulated during OX phase, consistent with the concept that it represents a temporal window devoted to growth. Interestingly, many genes involved in various aspects of RNA processing and degradation are also highly up-regulated during the OX phase (Tu et al. 2005). Furthermore, many mRNA transcripts are at their lowest levels toward the end of OX phase. These observations suggest that many transcripts are actively being degraded during OX phase, perhaps in response to oxidative damage or in preparation for cell division.

The RB (reductive, building) phase follows the $\mathrm{OX}$ phase and is characterized by a significant decrease in the rate of oxygen consumption (Fig. 1). During the midst of RB phase, oxygen consumption almost entirely ceases, which is marked by a sudden spike in dissolved oxygen levels. During this phase, the overwhelming majority of genes encoding mitochondrial proteins are up-regulated ( Tu et al. 2005). The collective up-regulation of these mitochondrial gene products suggests that cells might be rebuilding their mitochondria during this reductive temporal window, perhaps in response to the previous period of intense respiration in the OX phase. Furthermore, many genes that have functions associated with cell division are also up-regulated during $\mathrm{RB}$ phase (Tu et al. 2005). These gene products include histones, spindle pole body components, and gene products known to be important for the initiation of cell divison. As predicted by the microarray data, the initiation of cell division during the YMC is strictly gated to this reductive RB phase, as normal wild-type cells are never observed to divide during $\mathrm{OX}$ phase ( $\mathrm{Tu}$ et al. 2005). Approximately $40-50 \%$ of cells enter the cell cycle in each metabolic cycle (Tu et al. 2005).

Interestingly, cell cycle mutants that are forced to partake in cell division during the OX phase of the YMC were found to display higher spontaneous mutation rates (Chen et al. 2007). These observations suggest that restriction of DNA replication and cell division to the reductive phases serves to minimize oxidative damage to DNA, thereby reducing the rate of spontaneous mutation (Tu et al. 2005; Chen et al. 2007). Restriction of DNA replication to a reductive metabolic environment was also found to occur during short-period, 40-minute yeast oscillations (Klevecz et al. 2004). The gating of the cell cycle by the YMC might be formally analagous to gating of the cell cycle by the circadian cycle, first observed in cyanobacteria and later in mammals (Mori et al. 1996; Kondo et al. 1997; Matsuo et al. 2003; Nagoshi et al. 2004). These findings provide substantial evidence that there has been selective pressure to confine DNA replication to optimal temporal windows. Overall, the RB phase is associated with the induction of many mitochondrial and cell cycle genes during a period of lower oxygen consumption.

The RC (reductive, charging) phase is the longest of the three phases and immediately follows RB phase (Fig. 1). During this temporal window, many gene products that specify functions associated with nonrespiratory modes of metabolism are up-regulated (Tu et al. 2005), including peroxisomal proteins, enzymes involved in the storage and breakdown of carbohydrates, and enzymes necessary for ethanol utilization. The predicted outcome of these reactions is the production of acetyl-CoA units, which is the substrate for respiration, a hallmark of the impending OX phase. These observations indicate that during RC phase, yeast cells become dependent on these additional metabolic strategies for energy production and preparation for OX phase.

Many genes associated with starvation and stress-associated responses are also highly up-regulated during RC phase (Tu et al. 2005), including components of the vacuole, proteasome, and ubiquitination machinery and genes required for autophagy. In addition, many heat shock protein (HSP) genes are also highly induced. These observations predict the increased and regulated turnover of proteins and organelles during $\mathrm{RC}$ phase. Thus, this RC temporal window appears to be dedicated to the rebuilding and recharging of the cell, perhaps in preparation for the subsequent OX phase and a new round of growth. As such, vacuole-mediated catabolism and autophagy might in fact occur normally during the life of a yeast cell under nutrient-poor growth conditions.

\section{PREDICTION AND ANNOTATION OF GENE FUNCTION BASED ON YMC EXPRESSION PROFILES}

The temporal expression pattern of a gene during the YMC can often provide insights into the biological function of the encoded protein. For example, the open reading frame YHR075c (PPE1 or MRPS2) was previously identified to encode a mitochondrial ribosomal protein of the small subunit. Upon alignment of the expression patterns of all known genes encoding components of the mitochondrial ribosomes, all of them displayed highly similar temporal expression patterns except for PPE1, which peaks at a time distinct from that of the others $(\mathrm{Tu}$ et al. 2005). Subsequent studies have shown that PPE1 instead encodes a protein with carboxyl methyl esterase activity and might have been misannotated as a mitochondrial ribosomal protein (Wu et al. 2000). 
As a second example, the temporal expression profiles of periodic heat shock protein genes $(H S P)$ reveals that two of them (HSP10, HSP60) are expressed maximally in the RB phase, whereas the majority of others peak during the RC phase (Tu et al. 2005). Hsp10p and Hsp60p are localized to the mitochondria which is entirely consistent with their expression peaks in the RB phase, which is when other mitochondria gene products tend to be induced. Thus, a simple phase assignment based on expression during the YMC can be useful in predicting the function of uncharacterized genes.

Inspection of the temporal expression profiles of the YMC reveals that genes having a common function occasionally exhibit slightly distinct moments of expression, most notably cell cycle genes. Using a deconvolutionbased algorithm, the precise peaks of expression of cell cycle transcriptionally regulated genes were timed to a resolution of about 2 minutes (Rowicka et al. 2007). These data have presented a landscape of the transcriptional events that occur during the yeast cell cycle at a remarkably fine level of detail, and they reveal that many genes are transcribed just when their protein product is needed (a phenomenon termed "just-in-time synthesis"). Future application of such algorithms will provide additional insights into the precision with which cells have temporally optimized their transcriptional output.

\section{BEYOND TRANSCRIPTION}

The periodic transcription of genes during the YMC will undoubtedly direct many periodic outputs in the cell. In addition to respiration, cell division, and mitochondrial homeostasis, numerous other fundamental processes are predicted to be temporally regulated by the YMC. As an example, electron microscopic analysis of cells isolated during different temporal windows of the YMC have demonstrated that the vacuole exhibits dynamic changes in morphology during metabolic oscillation ( $\mathrm{Tu}$ et al. 2005). During the late RC and Ox phases, the vacuole becomes more prominent and markers of autophagy become visible. These observations suggest that autophagy and vacuole-mediated catabolism might be normal aspects of the life cycle of a cell under nutrient-poor growth conditions.

The many oscillating gene expression patterns predict that the YMC should differentially control metabolic state (Tu and McKnight 2006). Certain metabolites might exhibit periodic fluctuation and, in turn, have a reciprocal role in regulating the YMC. To determine whether cyclic changes in metabolic state might occur during the YMC, both liquid chromatography-tandem mass spectrometry (LC-MS/MS) and two-dimensional gas chromatography/time-of-flight mass spectrometry (GCxGC-TOFMS) metabolite profiling methods were used to monitor the intracellular concentrations of common metabolites at different time points of the cycles (Tu et al. 2007). The results of these surveys show that many metabolites, including amino acids, nucleotides, and carbohydrates, oscillate in abundance with a periodicity precisely matching that of the YMC (Tu et al. 2007). From analysis of these metabolite profiling data, the logic of metabolite oscillation largely matches that predicted by the YMC transcript data set. Moreover, the metabolite profiling extends this logic in ways not inherently obvious by inspection of gene expression profiles. These results imply that many cellular processes will be intimately coupled to these cyclic changes in a metabolic or redox state and thereby executed more optimally and efficiently.

\section{SIMILARITIES BETWEEN THE YMC AND CIRCADIAN CYCLE}

The circadian cycle temporally orchestrates many aspects of organismal metabolism and physiology (Rutter et al. 2002; Lowrey and Takahashi 2004). Genome-wide expression studies reveal that the circadian clock drives the periodic expression of many genes known to control metabolic state, including those that encode the rate-limiting enzymes of numerous metabolic processes (Harmer et al. 2000; Claridge-Chang et al. 2001; McDonald and Rosbash 2001; Panda et al. 2002). In mice, the expression of mitochondrial oxidative phosphorylation genes in the suprachiasmatic nucleus (SCN) oscillates in a circadian fashion (Panda et al. 2002), which indicates that respiration might be periodically up-regulated in a manner similar to the periodic bursts of respiration that are a hallmark of the YMC. The uptake of 2-deoxyglucose is known to be robustly periodic as a function of the day/night cycle in the SCN (Schwartz and Gainer 1977). Such data indicate that fundamental metabolic processes in SCN neurons fluctuate as a function of the circadian cycle.

Less well understood is the cyclic relationship between metabolism and circadian rhythm, whereby metabolism can, in turn, directly feed back to entrain rhythm. Restricted feeding can reset the phase of circadian gene expression in the liver, which demonstrates that food is a potent zeitgeber for the peripheral oscillators (Damiola et al. 2000; Stokkan et al. 2001). Moreover, several studies have suggested that small-molecule metabolites can directly modulate the activity of core clock components (Rutter et al. 2001; Dioum et al. 2002). However, the precise biochemical mechanisms by which food entrainment occurs remain poorly understood.

These numerous connections between metabolism and the circadian cycle parallel the cardinal properties of the YMC (Tu and McKnight 2006). In both cycles, the periodic expression of genes directs a variety of cellular and metabolic outputs. Both cycles can, in turn, be modulated by feeding conditions and metabolism. A preliminary analysis revealed that among the circadian periodic genes of Drosophila melanogaster that encode proteins with clear homologs in yeast, most of these yeast genes were also highly periodic during the YMC (Table 1). Such correlative observations might be indicative of either evolutionary conservation of periodic gene expression or a convergent solution to the same biological challenge.

Much like the YMC, the circadian cycle thus has a key role in optimizing cellular and metabolic output via temporal compartmentalization. In turn, metabolic state will likely alternate during the course of each approximately 
Table 1. Circadian Periodic Genes in Drosophila and Their Yeast Homologs

\begin{tabular}{lllr}
\hline Drosophila & Yeast & Function & $\begin{array}{c}\text { Periodicity } \\
\text { score }(\sigma)\end{array}$ \\
\hline Fdxh & YAH1 & iron-sulfur protein homologous to human adrenodoxin & 4.52 \\
HSP26 & HSP26 & heat shock protein 26 & 4.34 \\
cat & CTA1 & catalase A & 4.34 \\
INO-1 & INO1 & L-myo-inositol-1-phosphate synthase & 4.10 \\
CG9748 & DBP1 & putative ATP-dependent RNA helicase, DEAD box protein & 4.02 \\
CG7828 & ULA1 & required for activation of RUB1 (neddylation) & 3.95 \\
Septin1 & CDC11 & component of septin ring required for cytokinesis & 3.59 \\
Nsf2 & SEC18 & involved in protein transport between ER and Golgi & 3.12 \\
CG6145 & YEL041W & strong similarity to Utrlp & 3.11 \\
GC8468 & MCH5 & similarity to human X-linked PEST-containing transporter & 3.04 \\
Cyp4d21 & ERG11 & cytochrome P450 lanosterol 14a-demethylase & 3.04 \\
CG3021 & SLM3 & tRNA-specific 2-thiouridylase & 2.99 \\
Pdh & YMR226C & similarity to ketoreductases & 2.75 \\
CG4963 & MRS4 & mitochondrial iron transporter & 2.52 \\
CG7288 & SAD1 & zinc finger protein involved in pre-mRNA splicing & 0.93 \\
moira & SWI3 & transcription factor & 0.54 \\
zw & ZWF1 & glucose-6-phosphate dehydrogenase & -0.57 \\
\hline
\end{tabular}

Of the approximately 158 genes that are expressed periodically as a function of the circadian cycle in the fly head (Claridge-Chang et al. 2001), 17 have clear yeast homologs. Of these, 14 of 17 are highly periodically expressed $(>2 \sigma)$ as a function of the YMC. (We thank A. Kudlicki and M. Rowicka for assistance in the preparation of this table.)

24-hour period and feed back to modulate the activity of the circadian machinery. These considerations form an explicit prediction that the circadian cycle is, most fundamentally, a metabolic cycle (Rutter et al. 2002; Tu and McKnight 2006).

\section{OSCILLATIONS IN COMMON LABORATORY STRAINS OF YEAST?}

The prototrophic, genetically tractable CEN.PK strain, first adopted by the European yeast community (van Dijken et al. 2000), is our strain of choice for the study of these robust, approximately 4-5-hour metabolic cycles. Do common laboratory strains, such as the S288C and W303 strain backgrounds, also exhibit metabolic cycles? These popular yeast strains have been domesticated and contain mutations in multiple metabolic genes that serve as selectable nutritional markers. Although these auxotrophic markers have greatly facilitated the development of genetic screens and tools, they might compromise the output of cellular and metabolic pathways in ways not inherently obvious and elicit compensatory responses that are not typical of wild strains of yeast. For example, a laboratory strain that cannot synthesize adenine, uracil, or methionine will be absolutely dependent on supplementation of these metabolites for growth, which will undoubtedly alter flux through various biosynthetic pathways and hence the regulation of particular metabolic processes. Thus far, even using the parental S288C prototrophic strain, we have only observed low-amplitude oscillations of a period of about 80 minutes (B. Tu, unpubl.), which seem quite different from the robust approximately 4-5hour cycles exhibited by the prototrophic CEN.PK strain. Thus, we speculate that many common laboratory strains have been compromised in their ability to oscillate, specifically due to domestication and the presence of auxotrophic nutritional markers.

\section{LOG-PHASE, NUTRIENT-RICH GROWTH VS. CONTINUOUS, NUTRIENT-POOR CHEMOSTAT GROWTH}

How do the chemostat growth conditions used to observe the YMC compare to traditional log-phase growth conditions? Although the use of log-phase growth conditions is convenient and often informative, they might not always be representative of the conditions encountered by yeast in the wild. Yeast typically grow in colonies, where the cell density is very high and the distance between cell neighbors is very small. In the wild, yeast colonies will likely be exposed to environments where nutrients are poor, unlike the nutrient-rich conditions of log phase. Thus, the dense population of cells in the chemostat that undergo the YMC might approximate a colony exposed to a nutrient-poor environment. Under these challenging conditions, yeast cells deploy a more extensive assortment of regulatory strategies that are not typical of laboratory strains grown at log phase. Under nutrient-rich growth conditions, yeast tend not to perform mitochondrial respiration. Likewise, many classes of genes, including those encoding products required for respiration and fatty acid oxidation, are minimally expressed in $\log$ phase (Ghaemmaghami et al. 2003). In contrast, almost all genes required for mitochondrial and peroxisomal function are not only expressed, but also coordinately activated during precise temporal windows of the YMC (Tu et al. 2005). Thus, the growth conditions (i.e., high cell density and nutrient-limited) used to observe the YMC are perhaps more representative of what yeast cells encounter in the wild. Moreover, the use of chemostats for yeast growth enables the maintenance of constant $\mathrm{pH}$, temperature, aeration, and nutrient levels, thereby ensuring continuous, homogeneous growth conditions that are not easily achievable by use of batch cultures grown under high-glucose conditions. In doing so, the use of chemostats has enabled the observation of aspects of the 
life of a yeast cell that would otherwise be either obscure or not even utilized. Indeed, as the famous physicist Leo Szilard, inventor of the chemostat, once predicted: "a study of this slow-growth phase by means of the chemostat promises to yield information of some value on metabolism, regulatory processes, adaptations, and mutations of microorganisms." (Novick and Szilard 1950)

\section{CONCLUSIONS}

In summary, the yeast metabolic cycle is composed of an intricate transcriptional program that specifies a variety of periodic cellular outputs during the life of a yeast cell. In doing so, many fundamental cellular and metabolic processes are precisely compartmentalized in time, enabling cells to optimize metabolic output and improve fitness under nutrient-poor growth conditions. As a consequence of temporal compartmentalization, cyclic changes in metabolic state occur during the YMC, which might have an essential reciprocal role in the establishment and maintenance of metabolic oscillation. The numerous similarities between the YMC and circadian cycle suggest that certain principles of temporal orchestration might be conserved across other biological cycles and further indicate that the circadian cycle can be viewed as fundamentally a metabolic cycle. It is hopeful that future studies of the yeast metabolic cycle will continue to contribute to our understanding of the mechanisms of temporal compartmentalization and the basis of the circadian and other biological cycles.

\section{ACKNOWLEDGMENTS}

The authors thank funding support from a National Institutes of Health Director's Pioneer Award (S.L.M.), unrestricted funds from an anonymous donor (S.L.M.), a Helen Hay Whitney Foundation postdoctoral fellowship (B.P.T.), a Sara \& Frank McKnight Foundation Fellowship (B.P.T.), and a Burroughs Wellcome Fund Career Award in Biomedical Sciences (B.P.T.).

\section{REFERENCES}

Chance B., Estabrook R.W., and Ghosh A. 1964. Damped sinusoidal oscillations of cytoplasmic reduced pyridine nucleotide in yeast cells. Proc. Natl. Acad. Sci. 51: 1244.

Chen Z., Odstrcil E.A., Tu B.P., and McKnight S.L. 2007. Restriction of DNA replication to the reductive phase of the metabolic cycle protects genome integrity. Science 316: 1916.

Claridge-Chang A., Wijnen H., Naef F., Boothroyd C., Rajewsky N., and Young M.W. 2001. Circadian regulation of gene expression systems in the Drosophila head. Neuron 32: 657.

Damiola F., Le Minh N., Preitner N., Kornmann B., Fleury-Olela F., and Schibler U. 2000. Restricted feeding uncouples circadian oscillators in peripheral tissues from the central pacemaker in the suprachiasmatic nucleus. Genes Dev. 14: 2950.

Dioum E.M., Rutter J., Tuckerman J.R., Gonzalez G., GillesGonzalez M.A., and McKnight S.L. 2002. NPAS2: A gasresponsive transcription factor. Science 298: 2385.

Ghaemmaghami S., Huh W.K., Bower K., Howson R.W., Belle A., Dephoure N., O'Shea E.K., and Weissman J.S. 2003. Global analysis of protein expression in yeast. Nature 425: 737. Harmer S.L., Hogenesch J.B., Straume M., Chang H.S., Han B., Zhu T., Wang X., Kreps J.A., and Kay S.A. 2000.
Orchestrated transcription of key pathways in Arabidopsis by the circadian clock. Science 290: 2110.

Klevecz R.R., Bolen J., Forrest G., and Murray D.B. 2004. A genomewide oscillation in transcription gates DNA replication and cell cycle. Proc. Natl. Acad. Sci. 101: 1200.

Kondo T., Mori T., Lebedeva N.V., Aoki S., Ishiura M., and Golden S.S. 1997. Circadian rhythms in rapidly dividing cyanobacteria. Science 275: 224.

Lowrey P.L. and Takahashi J.S. 2004. Mammalian circadian biology: Elucidating genome-wide levels of temporal organization. Annu. Rev. Genomics Hum. Genet. 5: 407.

Matsuo T., Yamaguchi S., Mitsui S., Emi A., Shimoda F., and Okamura H. 2003. Control mechanism of the circadian clock for timing of cell division in vivo. Science 302: 255.

McDonald M.J. and Rosbash M. 2001. Microarray analysis and organization of circadian gene expression in Drosophila. Cell 107: 567.

Mori T., Binder B., and Johnson C.H. 1996. Circadian gating of cell division in cyanobacteria growing with average doubling times of less than 24 hours. Proc. Natl. Acad. Sci. 93: 10183.

Nagoshi E., Saini C., Bauer C., Laroche T., Naef F., and Schibler U. 2004. Circadian gene expression in individual fibroblasts: Cell-autonomous and self-sustained oscillators pass time to daughter cells. Cell 119: 693.

Novick A. and Szilard L. 1950. Description of the chemostat. Science 112: 715.

Panda S., Antoch M.P., Miller B.H., Su A.I., Schook A.B., Straume M., Schultz P.G., Kay S.A., Takahashi J.S., and Hogenesch J.B. 2002. Coordinated transcription of key pathways in the mouse by the circadian clock. Cell 109: 307.

Richard P. 2003. The rhythm of yeast. FEMS Microbiol. Rev. 27: 547 .

Rowicka M., Kudlicki A., Tu B.P., and Otwinowski Z. 2007. High-resolution timing of cell cycle gene expression. Proc. Natl. Acad. Sci. September 7.

Rutter J., Reick M., and McKnight S.L. 2002. Metabolism and the control of circadian rhythms. Annu. Rev. Biochem. 71: 307.

Rutter J., Reick M., Wu L.C., and McKnight S.L. 2001. Regulation of clock and NPAS2 DNA binding by the redox state of NAD cofactors. Science 293: 510.

Satroutdinov A.D., Kuriyama H., and Kobayashi H. 1992. Oscillatory metabolism of Saccharomyces cerevisiae in continuous culture. FEMS Microbiol. Lett. 77: 261.

Schwartz W.J. and Gainer H. 1977. Suprachiasmatic nucleus: Use of 14C-labeled deoxyglucose uptake as a functional marker. Science 197: 1089.

Stokkan K.A., Yamazaki S., Tei H., Sakaki Y., and Menaker M. 2001. Entrainment of the circadian clock in the liver by feeding. Science 291: 490.

Tu B.P. and McKnight S.L. 2006. Metabolic cycles as an underlying basis of biological oscillations. Nat. Rev. Mol. Cell Biol. 7: 696.

Tu B.P., Kudlicki A., Rowicka M., and McKnight S.L. 2005. Logic of the yeast metabolic cycle: Temporal compartmentalization of cellular processes. Science 310: 1152.

Tu B.P., Mohler R.E., Liu J.C., Dombek K.M., Young E.T., Synovec R.E., and McKnight S.L. 2007. Cyclic changes in metabolic state during the life of a yeast cells. Proc. Natl. Acad. Sci. October 16.

van Dijken J.P., Bauer J., Brambilla L., Duboc P., Francois J.M., Gancedo C., Giuseppin M.L., Heijnen J.J., Hoare M., Lange H.C., Madden E.A., Niederberger P., Nielsen J., Parrou J.L., Petit T., Porro D., Reuss M., van Riel N., Rizzi M., Steensma H.Y., Verrips C.T., Vindelov J., and Pronk J.T. 2000. An interlaboratory comparison of physiological and genetic properties of four Saccharomyces cerevisiae strains. Enzyme Microb. Technol. 26: 706.

von Meyenburg H.K. 1969. Energetics of the budding cycle of Saccharomyces cerevisiae during glucose limited aerobic growth. Arch. Microbiol. 66: 289.

Wu J., Tolstykh T., Lee J., Boyd K., Stock J.B., and Broach J.R. 2000 . Carboxyl methylation of the phosphoprotein phosphatase $2 \mathrm{~A}$ catalytic subunit promotes its functional association with regulatory subunits in vivo. EMBO J. 19: 5672. 


\section{$\overbrace{\text { CSH' }}^{\infty}$ Cold Spring Harbor Symposia SYMPOSIA}

\section{The Yeast Metabolic Cycle: Insights into the Life of a Eukaryotic Cell}

B. P. Tu and S. L. McKnight

Cold Spring Harb Symp Quant Biol 2007 72: 339-343

Access the most recent version at doi:10.1101/sqb.2007.72.019

References This article cites 27 articles, 15 of which can be accessed free at: http://symposium.cshlp.org/content/72/339.full.html\#ref-list-1

\section{License} Email Alerting
Service
top right corner of the article or click here. 